Thomas S. Ruta MD,' John C. Drummond mD, ${ }^{2}$ Daniel J. Cole $M^{3}$

\title{
A comparison of the area of histochemical dysfunction after focal cerebral ischaemia during anaesthesia with isoflurane and halothane in the rat
}

The investigations that have thus far evaluated the cerebral protective properties of isoflurane have provided conflicting results. Protection would be most likely 10 occur in the circumstances of incomplete cerebral ischaemia in which there is a penumbral zone of marginal perfusion. The present investigation sought to evaluate further the protective properties of isoflurane in that circumstance. Middle cerebral artery occlusion (MCAO) was performed in Sprague-Dawley rats anaesthetized with 1.2 MAC concentrations of either halothane or isoflurane. At the end of four hours of MCAO, the brains were removed, sectioned and incubated in the histochemical stain 2-3-triphenyltetrazolium (TTC). An image analysis system was used to measure the area of reduced or absent TTC staining in four coronal planes spanning the distribution of the middle cerebral artery. There was no difference benveen halothane and isoflurane anaesthetized animals with respect to the area of brain with evidence of histochemical dy.function. It is con-

\section{Key words}

ANAESTHETICS, VOLATILE: halothane, isoflurane; BRAIN: blood flow, protection, ischaemia.

From the Departments of Anaesthesia, University of Manitoba, Winnipeg, Manitoba, Canada R3E 1X2, ${ }^{1}$ University of California, San Diego; VA Medical Center, San Diego, California $92093,{ }^{2}$ and Loma Linda University School of Medicine, Loma Linda, California $92350 .^{3}$

The investigation was carried out in the Department of Anesthesiology of the VA Medical Center, San Diego, California, and was supported by the Department of Veterans Affairs.

Address correspondence to: Dr. J.C. Drummond, Neuroanesthesia Research, Mailstop-0629, UCSD, La Jolla CA, 92093.

Accepted for publication 14 September, 1990. cluded that isoflurane is not protective (relative to the status of halothane anaesthetized control animals) when administered at 1.2 MAC concentration during four hours of focal (incomplete) cerebral ischaemia in the rat.

Les investigations faites à date pour évaluer les propriétés protectrices cérébrales de l'isoflurane ont fourni des résultats divergents. La protection surviendrait le plus probablement dans les circonstances où l' ischémie cérébrale est incomplète et qui serait accompagnée en périphérie d' une zone où la perfusion est marginale. La présente investigation tente d'évaluer les propriétés protectrices' de l'isoflurane dans ces circonstances. L'occlusion de l'artère cérébrale mojenne (MCAO) a été faite chez des rats Sprague-Dawley anesthésiés avec des concentrations de 1,2 MAC soit de l'halothane, soit de l'isoflurane. A la fin de la quatrième heure de $M C A O$, le cerveau fut extrait, sectionné et incubé avec un colorant histochimique de 2-3. rriphenyltetrazolium (TTC). Le système d'analyse de l'image a été utilisé afin de mesurer la région de la réduction ou de l'absence de la coloration TTC dans quatre plans de section rayonnant à partir de l'artère cérébrale moyenne. II n'y avait aucune différence entre les animaux anesthésiés à l' halothane et l'isoflurane quant à la région du cerveau qui a démontré une évidence de dysfonction histochimique. On conclut qua l'isoflurane n'amène pas de protection (comparativement aux animaux contrôle anesthésiés à l'halothane) quand administré à des concentrations de 1,2 MAC lors d'une ischémie cérébrale focale (incomplète) de quatre heures chez les rats.

There is a risk of focal cerebral ischaemia during a variety of procedures performed under anaesthesia. As a result, there has been considerable interest in identifying anaesthetic agents that have the potential to protect the brain during cerebral ischaemia. Barbiturate anaesthesia can produce substantial suppression of cerebral metabolic rate 
and has been shown to provide cerebral protection. ${ }^{1,2}$ However, barbiturate administration is associated with cardiovascular depression and a prolonged time to wakeup. Isoflurane is a volatile inhalational agent which, in clinically relevant concentrations, produces a degree of cerebral metabolic depression equivalent to that attainable with barbiturates. ${ }^{3}$ However, the anaesthetic effects of isoflurane are readily reversed and the time to wake-up is not prolonged. There have been numerous investigations of the cerebral protective potential of isoflurane. However, the results have been inconsistent. In spite of one provocative retrospective study that indicated that, in man, the CBF threshold for the appearance of the EEG signs of ischaemia is lower during anaesthesia with isoflurane than with other inhaled agents, ${ }^{4}$ the bulk of the experimental data derived from prospective investigations that have examined neurological and/or histological endpoints do not confirm the protective effect of isoflurane..$^{5-8}$ The present study was undertaken in an attempt to provide additional laboratory confirmation of the protective potential of isoflurane. It compares the extent of histochemical dysfunction (as assessed with 2,3,5-triphenyltetrazolium chloride ${ }^{y-13}$ (TTC) occurring after four hours of focal cerebral ischaemia during anaesthesia with isoflurane or halothane. Halothane was selected as the "control" anaesthetic because it causes less depression of cerebral metabolic rate (CMRO2) than equi-MAC concentrations of isoflurane, and is not commonly viewed as a cerebral protectant. The model employed (middle cerebral artery occlusion (MCAO) in the rat) is one of incomplete cerebral ischaemia. It was chosen because it is probable that it is in circumstances wherein there is a zone of marginal cerebral perfusion in which the failure of energy supply and membrane function is gradual (cf. the rapid, complete failure of energy supply associated with complete cerebral ischaemia, eg., cardiac arrest) that the protective effects related to metabolic suppression or favourable $\mathrm{CBF}$ redistribution are most likely to be manifest.

\section{Methods}

The protocol was reviewed and approved by the Animal Use Subcommittee of the VA Medical Center. Twentysix male Sprague-Dawley rats (Harlan, Indianapolis, IN) weighing $350-450 \mathrm{~g}$ were fasted overnight and were allowed unrestricted access to water. On the day of study, the animals were assigned alternately to either the isoflurane $(n=13)$ or halothane $(n=13)$ anaesthetic groups. The animals were anaesthetized in a plexiglass induction chamber with either four per cent isoflurane or three per cent halothane in oxygen. Following induction of anaesthesia, the trachea was intubated and the lungs were mechanically ventilated with $3 \mathrm{~cm}$ of added PEEP.
The tidal volume and rate remained constant and the inspired carbon dioxide concentration was adjusted to maintain normocarbia $\left(\mathrm{PaCO}_{2} 35-40 \mathrm{mmHg}\right)$. A single dose of pancuronium, $0.2 \mathrm{mg}$, was administered IV to facilitate surgical preparation. Anaesthesia was maintained with 1.2 MAC inspired concentrations of either isoflurane ( 1.9 per cent) or halothane ( 1.1 per cent) in 40 per cent oxygen/balance nitrogen. (MAC values ( $\% \pm$ $\mathrm{SD})$ for isoflurane and halothane in Sprague-Dawley rats, as previously determined in our laboratories, are $1.58 \pm$ 0.15 and $0.95 \pm 0.11$ respectively. ${ }^{15}$ ) Vaporizer output was confirmed prior to the start of each study with a mass spectrometer (Ohmeda 6000, multi-gas monitor, Ohmeda, Atlanta, GA). Rectal temperature was servo-controlled to $37^{\circ} \mathrm{C}$ with a heating pad and a heat lamp. One femoral artery and one femoral vein were cannulated with $\mathrm{PE}$ (polyethylene) 50 tubing. Wound margins were infiltrated with 0.25 per cent bupivacaine. The femoral artery was used for continuous measurement of mean arterial blood pressure (MAP), and for blood sampling for determination of arterial blood gases, plasma glucose concentration and haematocrit. The femoral vein was used for maintenance intravenous fluid administration (normal saline, 4 $\mathrm{ml} \cdot \mathrm{kg}^{-1} \cdot-\mathrm{l}$ ). MAP was maintained between 90-110 $\mathrm{mmHg}$. If MAP was less than $90 \mathrm{mmHg}$, a transfusion of 3-5 ml of whole donor blood was administered. If transfusion did not raise MAP to $90 \mathrm{mmHg}$ phenylephrine was infused IV. Any animal with a MAP greater than 110 $\mathrm{mmHg}$ at any time during the study period was excluded. Increments of sodium bicarbonate of $0.25 \mathrm{mEq}$ were administered IV as necessary to maintain arterial $\mathrm{pH}$ greater than 7.30 .

Following the vascular cannulations, a left subtemporal craniectomy was performed. The dura overlying the left middle cerebral artery (MCA) was reflected and the latter was occluded using microbipolar electrocautery under constant saline irrigation. ${ }^{16}$ The artery was coagulated between a point proximal to the olfactory tract and a sccond point at the intersection of the MCA with the inferior cerebral vein. Care was taken to avoid injury to the latter structure. The cauterized segment included the origin of the lenticulostriate branch. Following middle cerebral artery occlusion (MCAO) the head wound was closed with 4-0 Vicryl suture.

Anaesthesia was maintained with the original equiMAC concentrations of isoflurane or halothane for four hours following MCAO. Physiologic data (MAP, heart rate, arterial blood gases, haematocrit) were recorded every thirty minutes. Measurements made prior to and during MCAO were averaged to obtain representative values for the two periods. Plasma glucose determinations were performed prior to $\mathrm{MCAO}$ and four hours later prior to sacrifice. Changes in the rate of infusion of phenyl- 


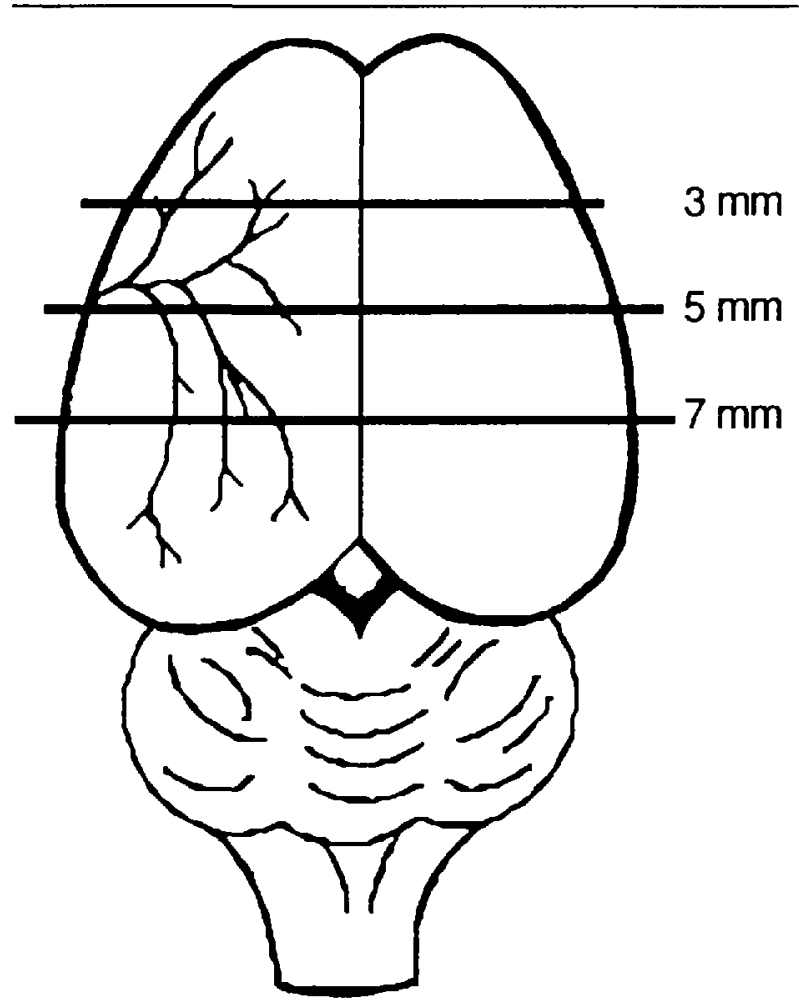

FIGURE Dorsal view of the rat brain showing the location of the threc coronal planes of section 3.5 and $7 \mathrm{~mm}$ posterior to the frontal poles, and the relation of those planes to the approximate distribution of the middle cerebral artery.

ephrine were noted as they occurred. The time during which phenylephrine was employed, and the average rate at which it was infused when required were calculated.

At the end of the four-hour period of MCAO, the animals were sacrificed by decapitation and the brains were removed rapidly. Each brain was sectioned coronally along planes 3,5 and $7 \mathrm{~mm}$ posterior the frontal pole (Figure). These sections, which span the distribution of the MCA, correspond approximately to Figures 17, 26, and 33 respectively in the atlas of the rat brain of Pelligrino et al. ${ }^{17}$ The four blocks of brain tissue that resulted were immersed promptly in a solution of two per cent TTC (A. Daigger \& Co, Wheeling, IL) in saline and were incubated at $37^{\circ} \mathrm{C}$ for 30 minutes. Immediately after incubation, photographs of the brain surfaces anterior and posterior to each of the three coronal planes of section were made using Ektachrome tungsten balanced ASA 60 colour slide film (Eastman Kodak, Rochester, NY). The photographs were subsequently analyzed using a Drexel/DUMAS image processing system (Drexel University Image Processing Center, Philadelphia, PA) to determine the area of brain with reduced or absent TTC staining. The portion of the hemisphere ipsilateral to MCAO in which reduced or absent staining was evident was expressed as a percentage of the total cross-sectional area of that hemisphere. In addition, in the same photographs, the cortex was analyzed separately and the portion that stained abnormally was expressed as a percentage of the total cross-sectional area of the entire ipsilateral cortex. The brain surfaces anterior and posterior to each coronal plane of section $(3,5$, and $7 \mathrm{~mm}$ posterior to the frontal pole) were analyzed. The data for the anterior and posterior surfaces of each plane were averaged to yield a single value for each region (entire hemisphere, and cortex alone) in each plane. Two observers blinded to the experimental course performed the image analysis independently. The measurements made by the two observers were averaged to obtain the reported values.

Between group comparisons of the physiological data and of the percentage area of brain with abnormal TTC staining were performed using $t$ tests for unpaired data. The data for the per cent arca of abnormal TTC staining were analyzed both with and without an arcsine transformation. The latter was performed because the use of percentages can result in a non-normal distribution when the data points are clustered near one boundary of the available range. Within group comparisons of plasma glucose levels were performed using paired t-tests. A $P$ value of less than 0.05 was considered significant.

\section{Results}

The weights (halothane group: $390 \pm 24 \mathrm{~g}$; isoflurane group: $401 \pm 34$ ) did not differ. The remaining physiological data are presented in Table 1 . There were no significant differences between groups for MAP, heart rate, $\mathrm{PaCO}_{2}, \mathrm{pH}$, haematocrit, and plasma glucose concentration prior to $\mathrm{MCAO}$. Prior to $\mathrm{MCAO}, \mathrm{PaO}_{2}$ in the isoflurane group $(143 \pm 32 \mathrm{mmHg})$ was significantly higher than in the halothane group (115 $\pm 23 \mathrm{mmHg}$ ). During MCAO heart rate and plasma glucose concentration were significantly lower in the halothane group. Plasma glucose was significantly increased in the isoflurane group four hours after MCAO but was unchanged in the halothane group.

There were no significant differences in terms of the time of infusion or the average rate of infusion of phenylephrine (Table I).

The data for the areas of brain with abnormal TTC staining are presented in Table II. In the three coronal planes examined, there were no significant differences (with or without arcsine transformation of the data) nor were any apparent trends for either the entire hemisphere or for cortex alone.

\section{Discussion}

The substantial cerebral metabolic suppression that occurs with clinical concentrations of isoflurane ${ }^{3}$ leads to 
TABLE I Physiological data (mean \pm SD) prior to (pre-MCAO) and during MCAO

\begin{tabular}{|c|c|c|c|c|}
\hline & \multicolumn{2}{|l|}{ Pre-MCAO } & \multicolumn{2}{|l|}{$M C A O$} \\
\hline & Halothane & Isoflurane & Halorhane & Isoflurane \\
\hline MAP* $(\mathrm{mmHg})$ & $94 \pm 7$ & $100 \pm 3$ & $95 \pm 3$ & $95 \pm 4$ \\
\hline Heart rate $(\mathrm{bpm})$ & $315 \pm 44$ & $344 \pm 28$ & $310 \pm 25$ & $345 \pm 26 \dagger$ \\
\hline $\mathrm{PaCO}_{2}(\mathrm{mmHg})$ & $37 \pm 2.3$ & $36 \pm 2.5$ & $38 \pm 2$ & $37 \pm 1$ \\
\hline $\mathrm{PaO}_{2}(\mathrm{mmHg})$ & $115 \pm 23$ & $143 \pm 32 \dagger$ & $141 \pm 26$ & $144 \pm 26$ \\
\hline $\mathrm{pH}$ & $7.39 \pm 0.03$ & $7.38 \pm 0.04$ & $7.36 \pm 0.02$ & $7.35 \pm 0.02$ \\
\hline Haematocrit (\%) & $44 \pm 3$ & $46 \pm 3$ & $43 \pm 1$ & $42=3$ \\
\hline $\begin{array}{l}\text { Plasma glucose } \\
\left(\mathrm{mg} \cdot \mathrm{dl}^{-1}\right)\end{array}$ & $96 \pm 35$ & $120 \pm 29$ & $117 \pm 27$ & $181 \pm 74 \div$ \\
\hline $\begin{array}{l}\text { Phenylephrine } \\
\left(\mu \mathrm{g} \cdot \mathrm{kg}^{-1} \cdot \min ^{-1}\right)\end{array}$ & & & $1.4 \pm 0.7$ & $2.3 \pm 2.1$ \\
\hline $\begin{array}{l}\text { Duration of phenyl } \\
\text { Infusion (min) }\end{array}$ & & & $160 \pm 94$ & $91 \pm 92$ \\
\hline
\end{tabular}

*MAP indicates mean arterial pressure.

$\dagger P<0.05$ halothane vs isoflurane for pre-MCAO or MCAO data.

$\ddagger P<0.05$ pre-MCAO vs MCAO.

the reasonable supposition that it might have cerebral protective properties in the setting of focal (incomplete) cerebral ischaemia. Only one relevant investigation has been performed in man and it provided support for this notion. Michenfelder et al. ${ }^{4}$ analyzed the EEG changes that occurred in patients undergoing carotid endarterectomy performed during various anaesthetic regimens. They observed that, during the period of internal carotid artery occlusion, the "critical" cerebral blood flow (the CBF above which EEG changes suggestive of cerebral ischaemia were not observed in the majority of patients) was lower during anaesthesia with isoflurane than with halothane or enflurane. However, that study, as acknowledged by the authors, was limited by the fact that it was retrospective and because the halothane and isoflurane groups were non-concurrent.

The limitations of the single human investigation mentioned above have resulted in a considerable interest in establishing definitive laboratory confirmation of the protective effect of isoflurane. However, the bulk of the relevant animal investigations, including the present study, has not provided support for a protective effect of isoflurane. Nehls et al. performed six hours of middle cerebral artery occlusion in baboons anaesthetized with isoflurane, barbiturate or fentanyl $/ \mathrm{N}_{2} \mathrm{O} .{ }^{6}$ They observed a better neurological and histological outcome in the barbiturate group and accordingly could not confirm the protective effect of isoflurane. That study, however, was criticized because of differences between the groups in blood pressure and pressor doses. Milde et al. examined neurological and neuropathological outcome in monkeys that underwent five hours of MCAO during anaesthesia with isoflurane or barbiturate. ${ }^{18}$ They found no difference between groups. That study, however, is also subject to criticism in that there was no control group to confirm the sensitivity of the model. Warner et al. ${ }^{5}$ compared $\mathrm{N}_{2} \mathrm{O}$ sedated and isoflurane anaesthetized rats and found no difference in neuropathological outcome after ten minutes of forebrain ischaemia (bilateral carotid artery occlusion with simultaneous hypotension). Gelb et al. ${ }^{8}$ induced hypotension ( $\mathrm{MAP} \approx 50 \mathrm{mmHg}$ ) in monkeys during a 45 minute period of MCAO. The induced hypotension was accomplished with either sodium nitroprusside superimposed on an anaesthetic of 0.75 per cent halothane or with isoflurane alone (average end-tidal isoflurane concentration ( \pm SD) during hypotension: $1.9 \pm 0.7$ per cent). They found no differences in neurological or histological outcome. Baughman et al. ${ }^{7}$ compared the effects of isoflurane, halothane and $\mathrm{N}_{2} \mathrm{O}$ on outcome in rats after incomplete cerebral ischaemia (unilateral carotid artery occlusion with haemorrhagic hypotension) of moderate and severe degrees. They reported that neurological and histological outcome and mortality were worse among animals that received sedation with 70 per cent $\mathrm{N}_{2} \mathrm{O}$ than among those that received anaesthesia (1.0 MAC) with either halothane or isoflurane. However, there were no significant differences between animals anaesthetized with halothane or isoflurane.

The present study used the histochemical stain 2-3triphenyltetrazolium as its endpoint. TTC is a colourless compound that is converted to an intense red stain (formazan) in the presence of functioning mitochondrial oxidative enzyme systems. The failure of brain tissue to convert TTC to its coloured derivative has been reported to correlate well with histopathological outcome. ${ }^{9-13}$ However, there is some controversy as to whether a failure 
TABLE II The area (mean \pm SE) or reduced or absent TTC staining in the hemisphere (HEM) ipsilateral to MCAO (expressed as a per cent of total area of the hemispherc) and in the ipsilateral neocortex (CTX) (expressed as per cent of total ipsilateral cortical area) in coronal brain sections 3,5 , and $7 \mathrm{~mm}$ posterior to the frontal poles

\begin{tabular}{lllllll}
\hline Sectioniregion & $3-C T X$ & $3-H E M$ & $5-C T X$ & $5-H E M$ & $7-C T X$ & $7-H E M$ \\
\hline Isoflurane & $7.8 \pm 1.3$ & $9.2 \pm 2.3$ & $7.9 \pm 1.5$ & $12.2 \pm 1.8$ & $3.4 \pm 2.0$ & $4.2 \pm 2.0$ \\
Halothane & $6.0 \pm 1.4$ & $7.8 \pm 2.2$ & $8.2 \pm 1.3$ & $14.2 \pm 1.6$ & $1.6 \pm 0.8$ & $5.2 \pm 2.0$ \\
\hline
\end{tabular}

to stain with TTC invariably represents inevitable infarction, and there are data to indicate that at least some component of the histochemical dysfunction revealed by TTC may be reversible. ${ }^{14}$ Nonetheless, a deficiency of TTC staining indicates metabolic compromise and probably represents a reasonable basis for comparing the well being of brain tissue following a period of ischaemia. It was anticipated that the beneficial effects of isoflurane, if any, would be most likely to be manifest in the penumbral zone at the periphery of the ischaemic territory. It is in these regions of marginal perfusion (as opposed to densely ischaemic core areas with severe neuronal compromise) that a protective effect could favourably tip the balance between viability and imminent demise. Accordingly, it was anticipated that a protective effect would result in a reduction of the diameter the area of abnormal TTC staining. However, the analysis revealed no difference in the areas of reduced or abnormal TTC staining between animals anaesthetized with halothane and isoflurane.

In one phase of the present analysis, the neocortex was examined separately. This was done because it has been demonstrated that, while isoflurane in general causes a greater suppression of cerebral metabolic rate (CMR) than halothane, the differences are greater in the cortex than in sub-cortical structures. ${ }^{19}$ It might therefore be anticipated that the cerebral protective effects of isoflurane, if any, would be most obvious in cortex. It seemed feasible that, with a less selective analysis, cerebral protective effects might be obscured by the influences of damage to regions that were not subject to the protective effects because of differences in their CMR response to isoflurane. Nonetheless, selective protection of the cortex was not apparent.

There were differences in physiological variables between the two groups. During MCAO heart rate was significantly lower in the halothane group. This finding is consistent with properties described in standard references. ${ }^{20}$ As well, $\mathrm{PaO}_{2}$ was significantly less in the halothane group (115 $\pm 23 \mathrm{mmHg}$ ) than in the isoflurane group ( $143 \pm 32$ ) prior to MCAO but this difference was not apparent during MCAO. It is unlikely that these minor physiological differences significantly influenced the TTC results.
Plasma glucose concentrations also differed. The isoflurane group had a significantly greater glucose level after four hours of MCAO. This finding is consistent with other studies that have shown that isoflurane anaesthesia is associated with significantly higher plasma glucose levels than other inhaled agents in both man and animals. ${ }^{21,22}$ This phenomenon may be the result of a decreased insulin response to glucose. ${ }^{23}$ Numerous investigations have confirmed that hyperglycaemia increases neurological injury during CNS ischaemia. ${ }^{24-27}$ Therefore, it is possible that a protective effect of isoflurane may have been negated in the present study by the higher glucose levels in the isoflurane anaesthetized animals.

The present study evaluated the effects of a four-hour period of MCAO. It is possible that the protective potential of isoflurane will only be apparent in circumstances of near-threshold injury, and that the insult in the present experiment was too severe. The four-hour ischaemic time was chosen because of our preliminary observations that, in this species, the threshold for reliable development of a TTC deficit is approximately three hours. While the location of the salvageable periphery might well shift with increasing duration of occlusion, it seems probable that there would still be a marginally perfused region at the periphery of the ischaemic territory that would constitute a threshold injury state and that, in the event of some protective effect, the dimension of the TTC-deficient area would be influenced.

Our previous experience with this model has confirmed its capacity to identify a beneficial intervention. Measurement of the area of TTC deficiency has been employed in two previous investigations in our laboratories. ${ }^{14,28}$ In those studies, TTC measurements were made either three or five hours after vessel occlusion and in both, therapeutic interventions (induced hypertension ${ }^{28}$ and re-establishment of vessel patency ${ }^{14}$ ) were shown to diminish the size of the TTC lesion. We are nonetheless aware that the variability of TTC lesion size with this model is substantial and presents a limitation. For instance, the power of this model to confirm a 50 per cent reduction in lesion size at the centre of the ischaemic area is approximately $0.70 .^{29}$ However, the absence of even apparent trends toward a reduction of the area of injury in 
isoflurane anaesthetized animals (Table II) makes it unlikely that a substantial protective effect by isoflurane has been overlooked.

The concentration of isoflurane enployed, 1.2 MAC, may have been a factor. It is possible that this concentration is inadequate to provide protective effects, although previously available information leads to the expectation that it should be sufficient. The retrospective study of Michenfelder et al. of critical CBF during carotid endarterectomy ${ }^{4}$ and the small prospective investigation of Casement et al. ${ }^{27}$ indicated a protective effect for isoflurane in concentrations as low as 0.6 MAC. It has been surmised that the protective properties of isoflurane are the result of the suppression of CMR that it causes. A 1.2 MAC concentration of isoflurane is sufficient to cause a substantial suppression of CMR. In rats, the cerebral metabolic rate for glucose (CMRg) in the sensorimotor cortex is reduced to 44 per cent of awake control values by 1.0 MAC isoflurane anaesthesia, ${ }^{30}$ although additional reduction of CMRg does occur at higher concentrations. In addition, it has been demonstrated that, in this concentration range, isoflurane produces significantly greater metabolic depression than does 1.0 MAC halothane. In cats, a 1.0 MAC end-tidal concentration of isoflurane caused a 45 per cent reduction of CMRO2 (as compared with a nitrous oxide-sedated control state) while an equivalent MAC concentration of halothane caused a decrease of only 27 per cent. ${ }^{31}$ In rats, during $1.0 \mathrm{MAC}$ isoflurane anaesthesia, CMRg in the neocortex was 36 per cent less than that observed during 1.0 MAC halothane anaesthesia. ${ }^{19}$ Accordingly, it seems likely that the 1.2 MAC concentration employed in the present study should have been sufficient to provide any protective benefit related to CMR suppression.

Phenylephrine was used to support blood pressure to equivalent levels in the two anaesthetic groups. There were no statistical differences between the infusion rates or total duration of infusion of phenylephrine; however, there was substantial variation between animals. Nonetheless, the phenylephrine is unlikely to have influenced the results as it has been previously demonstrated in Sprague-Dawley rats during MCAO that phenylephrine does not result in cerebral vasoconstriction in either normal or ischaemic brain. ${ }^{32}$

In summary, the present study compared the effects of halothane and isoflurane on brain injury as measured by abnormal TTC staining after four hours of focal cerebral ischaemia in the rat. There were no significant differences in the size of the area of abnormal TTC staining. The present results do not provide support for the cerebral protective properties that have been attributed to isoflurane.

\section{Acknowledgements}

The authors are grateful for the technical assistance of Robin Giamela, M.S. and Joanne Steinauer. This work was supported by the Veterans Administration and The Canadian Heart and Stroke Foundation.

\section{References}

I Smith A, Hoff J. Nielsen S, Larson C. Barbiturate protection in acute focal cerebral ischemia. Stroke 1974; 5 : $1-7$.

2 Michenfelder J, Milde J, Sundt T. Ccrebral protection by barbiturate ancsthesia. Use after middlc cercbral antery occlusion in Java monkeys. Arch Ncurol 1976; 33: 345-50.

3 Newberg L, Milde J, Michenfelder J. The ccrebral metabolic effects of isofluranc at and above concentrations that suppress cortical electrical activity. Ancsthesiology 1983; 59: 23-8.

4 Michenfelder J, Sundt T, Fode N, Sharbrough F. Isoflurane when compared to enflurane and halothane decreases the frequency of cerebral ischemia during carotid endarterectomy. Anesthesiology 1987; 67: 336-40.

5 Warner $D$. Desphande J. Wieloch $T$. The effect of isoflurane on neuronal necrosis following ncar-complete forebrain ischemia in the rat. Anesthesiology 1986; 64: 19-23.

6 Nehls D. Todd M, Spetzler R, Drummond J. Thompson R, Johnson $P$. A comparison of the cercbral protective effects of isoflurane and barbiturates during temporary focal ischemia in primates. Anesthesiology 1987; 66: 453-64.

7 Baughman V, Hoffman W, Miletich D, Albrecht R. Thomas C. Neurologic outcome in rats following incomplete cerebral ischemia during halothane, isoflurane, or $\mathrm{N}_{2} \mathrm{O}$. Anesthesiology 1988; 69: 192-8.

8 Gelb A, Boisvert D. Tang $C$ et al. Primate brain tolerancc to temporary focal cerebral ischemia during isofluraneor sodium nitroprusside-induced hypotension. Anesthesiology 1989; 70: 678-83.

9 Bederson J, Pitts L, Germano S, Nishimura M, Davis R, Bartkowski $H$. Evaluation of 2,3,5-triphenyltetrazolium chloride as a stain for detection and quantification of expcrimental cerebral infarction in rats. Stroke 1986; 17: 1304-8.

10 Bose B, Jones $S$, Lorig R, Friel H, Weinstein W, Little J. Evolving focal cerebral ischemia in cats: spatial correlation of nuclear magnetic resonance imaging, cerebral blood flow, tetrazolium staining, and histopathology. Stroke 1988; 19: 28-37.

11 Liszczak T, Hedley-White E Adams $J$ er al. Limitation of tetrazolium salts in delineating infarcted brain. Acta Neuropathol 1984; 65: 150-7.

12 Lundy F, Solik B, Frank $R$ et al. Morphometric cvaluation of brain infarcts in rats and gerbils. J Pharmacol Meth 1986; 16: 201-14.

13 Park C. Mendelow A, Graham D, McCulloch J, Teasdale 
G. Correlation of triphenyltetrazolium chloride perfusion staining with conventional neurohistology in the detection of early brain ischacmia. Neuropathology and Applied Ncurobiology 1988; 14: 289-98.

14 Cole DJ, Drummond JC, Ghazal EA, Shapiro HM. A reversible component of cerebral injury as identified by the histochemical stain 2,3,5-triphenyltetrazolium chloride (TTC). Acta Neuropathol 1990; 80: 152-5.

15 Cole D, Shapiro H, Drummond J, Kalichman $M$. The nonlincar potency of sub-MAC concentrations of nitrous oxide in decreasing the anesthetic requircment of enflurane, halothane and isoflurane in the rat. Anesthesiology 1990; 73: 93-9.

16 Tamura A, Graham D, McCulloch J, Teasdale G. Focal cerebral ischacmia in the rat 1 . Description of technique and early neuropathological consequences following middle cerebral artery occlusion. J Cercb Blood Flow Metab 1981; 1: 53-60.

17 Pelligrino L, Pelligrino A, Cushman A. A stereotaxic atlas of the rat brain. New York; Plenum Press, 1979.

18 Milde L, Milde J, Lanier W, Michenfelder J. Comparison of the effects of isoflurane and thiopental on neurologic outcome and neuropathology after temporary focal cercbral ischemia in primates. Anesthesiology 1988; 69: 905-13.

19 Hansen $T$, Warner $D$. Todd $M$, Vust $L$. The role of cerebral metabolism in determining the local cerebral blood flow effects of volatilc anesthetics: evidence for persistent flow-metabolism coupling. J Cereb Blood Flow Mctab 1989; 9: 323-8.

20 Hickey RF, Eger EL. The circulatory pharmacology of inhaled anesthetics. I $n$ : Miller RD (Ed.). Anesthesia,. 2nd cd., New York: Churchill Livingstone Inc., 1986; 649-66.

21 Kofke W, Hawkins R, Davis D. BiebuyckJ. Comparison of the effects of volatile anesthetics on brain glucose metabolism in rats. Anesthesiology 1987; 66: 810-3.

22 Stevens $W$, Eger E, Joas $T$, Cromwell $T$, White A, Dolan $W$. Comparative toxicity of isoflurane, halothane, fluroxene, and diethyl ether in human volunteers. Can Anacsth Soc J 1973; 20: 357-68.

23 Diltoer $M$, Camo $F$. Glucose homcostasis and insulin secretion during isoflurane anesthesia in human. Anesthesjology 1988; 68: 880-6.

24 Pulsinelli W, Waldman S, Rawlinson D, Plum F. Moderate hyperglycemia augments ischemic brain damage: a neuropathologic study in the rat. Neurology 1982; 32 : 1239-46.

25 Lanier W, Stangland K, Scheithauer B, Milde J, Michenfelder $J$. The effects of dextrose infusion and hcad position on neurologic outcome after complete cerebral ischemia in primates: examination of a model. Anesthesiology 1987; 66: 39-48.
26 Drummond J, Moore $S$. The influence of dextrose administration on neurologic outcome after temporary spinal cord ischemia in the rabbit. Anesthesiology 1989; 70: 64-70.

27 Casement B, Messick J, Milde L, Sharbrough F. Michenfelder J. Sundt $T$. Critical rCBF during isoflurane anesthesia in man. Anesthesiology 1985; 63: A406.

28 Drummond JC, Oh Y-S, Cole DJ. Does phenylephrincinduced hypertension during focal cerebral ischemia aggravate brain cdema? Anesthesiology 1989; 71: AS67.

29 Glantz SA. Primer of Biostatistics. New York: McGrawHill, 1981, p. 146.

30 Maekawa T, Tommasino C, Shapiro H, Kiefer-Goodman J. Kohlenberger $R$. Local cerebral blood flow and glucose utilization during isoflurane anesthesia in the rat. Anesthesiology 1986; 65: 144-51.

31 Todd $M M$, Drummond JC. A comparison of the cerebrovascular and metabolic effects of halothanc and isoflurane in the cat. Anesthesiology 1984; 60;276-82.

32 Drummond JC, Oh Y-S, Cole DJ, Shapiro HM. Phenylephrine-induced hypertension decreases the area of ischemia following middle cerebral artery occlusion in the rat. Stroke 1989; 20: 1538-44. 